Abstract 176 Table 2 Cumulative comorbidities by year in caucasians compared to all other ethnicities

\begin{tabular}{|l|c|c|c|c|c|c|c|c|}
\hline & \multicolumn{7}{|c|}{ Follow up years since SLE diagnosis } \\
\hline Comorbidity & 1 & 2 & 3 & 4 & 5 & 6 & 7 & 8 \\
\hline AVE Caucasian (\%) & 0.59 & 0.88 & 2.06 & 2.95 & 3.54 & 4.13 & 4.42 & 5.60 \\
\hline AVE Other (\%) & 0.53 & 0.53 & 0.79 & 0.79 & 1.59 & 1.59 & 1.59 & 1.59 \\
\hline Osteoporosis Caucasians (\%) & 0.00 & 0.59 & 1.18 & 1.77 & 2.06 & 3.25 & 3.55 & 4.14 \\
\hline Osteoporosis Others (\%) & 0.79 & 0.79 & 0.79 & 1.06 & 1.06 & 1.06 & 1.32 & 1.32 \\
\hline Osteonecrosis Caucasians (\%) & 0.59 & 1.18 & 1.18 & 1.47 & 1.47 & 2.07 & 2.07 & 2.66 \\
\hline Osteonecrosis Others (\%) & 0.26 & 1.59 & 2.91 & 3.44 & 4.23 & 4.76 & 5.29 & 5.82 \\
\hline Diabetes Caucasian (\%) & 1.77 & 2.65 & 2.65 & 2.95 & 2.95 & 2.96 & 2.96 & 3.25 \\
\hline Diabetes Other (\%) & 1.85 & 2.12 & 2.12 & 2.38 & 2.38 & 2.91 & 3.17 & 3.70 \\
\hline
\end{tabular}

Results Of the 717 patients followed for at least 8 years, 90.2\% were female, $47.3 \%$ were Caucasian, $13.8 \%$ were Black, $19.4 \%$ were Asian, $16.3 \%$ Hispanic and 3.2\% other. Their mean age at enrolment was $34.2 \pm 13.1$ years and SLEDAI- $2 \mathrm{~K}$ at enrolment was $4.17 \pm 4.49$. The duration from diagnosis to enrolment was $5.9 \pm 4.4$ months.

Mean SDI gradually increases over 8 years. The accumulation of AVEs, osteoporosis, osteonecrosis and diabetes all increase progressively over an 8 year period. Caucasians accumulate AVEs and osteoporosis more frequently than all "other" ethnicities. In contrast, all "other" ethnicities accumulate osteonecrosis more frequently than Caucasians. All ethnicities accumulate diabetes at the same frequency.

Conclusions As expected disease damage and comorbidities in newly diagnosed patients increase over their first 8 years. Different ethnicities accumulate comorbidities at different rates.

\section{ASSOCIATION BETWEEN CHRONIC ANTIMALARIAL THERAPY AND ELEVATED MYOCARDIAL BIOMARKERS IN PATIENTS WITH SYSTEMIC LUPUS ERYTHEMATOSUS AND NO PRIOR HEART DISEASE: A PRELIMINARY REPORT}

${ }^{1} \mathrm{~K}$ Tselios, ${ }^{1} \mathrm{D}$ Gladman*, ${ }^{2} \mathrm{P}$ Harvey, ${ }^{1} \mathrm{~S} \mathrm{Su},{ }^{1} \mathrm{M}$ Urowitz. ${ }^{1}$ University of Toronto and Toronto Western Hospital, Rheumatology, Toronto, Canada; ${ }^{2}$ University of Toronto and Women's College Hospital, Medicine, Toronto, Canada

\subsection{6/lupus-2017-000215.177}

Background and aims Antimalarial (AM)-induced cardiomyopathy is an extremely rare complication of AM treatment in systemic lupus erythematosus (SLE). The use of specific cardiac biomarkers may identify patients at risk. We sought to investigate the prevalence and associated factors for abnormal myocardial biomarkers in lupus patients.

Methods Consecutive patients $(n=179)$ attending the Toronto Lupus Clinic were enrolled. BNP (brain natriuretic peptide, assessing pressure and/or volume overload) and cTnI (cardiac troponin I, assessing myocardial necrosis) were measured simultaneously. None had ECG abnormalities suggestive of acute coronary syndrome. Analysis was performed with SAS $9.3 ; \mathrm{p}<0.05$ was considered significant.
Results Twenty-seven patients (15.1\%) had elevated BNP and/ or cTnI; 11 with prior history of heart failure, coronary artery disease, pulmonary hypertension and/or exertional dyspnea were excluded. Compared to subjects with normal biomarkers, the remaining patients $(n=16)$ were older [54.7 \pm 15.1 vs. $47.8 \pm 12.2$ years, $p=0.037]$, had longer disease duration [22.6 \pm 10.4 vs. $15.5 \pm 10.1$ years, $\mathrm{p}<0.001]$, longer $\mathrm{AM}$ use $[12.5 \pm 9.6$ vs. $7.9 \pm 8$ years, $\mathrm{p}=0.034]$ and more frequently persistent CPK elevation [44.4 vs. $16.4 \%, \mathrm{p}<0.001]$. Multi-variable regression analysis showed chronic AM treatment combined with CPK elevation to be an important predictor for elevated myocardial biomarkers $[\mathrm{HR}=1.41$, 95\% $\mathrm{CI}=1.06-1.89, \mathrm{p}=0.02]$. Two patients were diagnosed with AM-induced cardiomyopathy on endomyocardial biopsy; both had CPK and BNP/cTnI elevation.

Conclusions Approximately 9\% of unselected SLE patients had elevated myocardial biomarkers, in the absence of prior cardiac disease. Chronic AM therapy accompanied by persistent CPK elevation conferred an increased risk for abnormal BNP and cTnI, which might predict cardiomyopathy in such patients.

\section{ASSESSMENT OF THE RISK OF FLARES IN SYSTEMIC LUPUS ERITHEMATOSUS}

${ }^{1} \mathrm{~L}$ Mazur-Nicorici*, ${ }^{1} \mathrm{~V}$ Sadovici-Bobeica, ${ }^{1} \mathrm{M}$ Garabajiu, ${ }^{2} \mathrm{~N}$ Revenco, ${ }^{1} \mathrm{M}$ Mazur. ${ }^{1}$ State Medical and Pharmaceutical University Nicolae Testemitanu, Department of Internal Medicine, Chisinau, Moldova; ${ }^{2}$ State Medical and Pharmaceutical University Nicolae Testemitanu, Department of Pediatry, Chisinau, Moldova

\subsection{6/lupus-2017-000215.178}

Background and aims A prospective analytic study was conducted during February 2015-February 2016, which included adult patients with SLE, according to SLICC, 2012 classification criteria. The subjects were prospectively fallowed-up every month 3, 5, 9, 12 visit and disease activity by SLEDAI and SLAM, SELENA/SLEDAI flare index and laboratory tests were assessed. The risk of flare was calculated by Pearson correlation coefficient and risk ratio (RR) with 95\% CI.

Results In the study were included 102 SLE patients, 94,1\% females, mean age \pm SD $42.4 \pm 13.3$ (range 20-73) years, mean disease duration \pm SD93.9 \pm 77.1 (range 0,1-228) months. 
Abstract 178 Table 1 Association of laboratory and clinical values (SLAM) with SLE flares

\begin{tabular}{lllll}
\hline & Correlation coefficient & $\mathbf{p}$ & RR & $95 \% \mathrm{Cl}$ \\
\hline ESR & 0.34 & $<0.05$ & 1.56 & $0.36-0.87$ \\
ANA & 0.22 & $>0.05$ & 1.12 & $0.43-1.50$ \\
AntiDNA Ab & 0.13 & $>0.05$ & 0.98 & $0.67-2,13$ \\
Low Hb level & 0.48 & $<0.05$ & 1.99 & $0.45-0.80$ \\
Low leucocytes & 0.23 & $>0.05$ & 1.01 & $0.56-1.33$ \\
Low lymphocytes & 0.56 & $<0.05$ & 2.05 & $0.33-0.67$ \\
Antiphospholipid syndrome & - & - & 2.30 & $0.61-0.88$ \\
Pulmonary Involvement & - & - & 1.88 & $0.23-0.82$ \\
\hline
\end{tabular}

During a 12 moths fallow-up, 55 flares were enregistered, including 11 cases of severe flares, with a SLEDAI increase from 3 to 17 points. So, the total incidence of flares was 0,53 patient/year and the incidence for severe flares was 0,10 patient/year. In order to assess the risk of flares, we have studied several potential risk factors, as shown in the table.

Conclusions the incidence of flare in a 12 months period was $53,9 \%$, including $10,8 \%$ of severe flares. Low $\mathrm{Hb}$ and lymphocytopenia are at risk for flares and antiphospholipid syndrome and pulmonary involvement were the main clinical risk factors in our cohort.

\section{BIOMARKERS OF ATHEROSCLEROSIS IN SLE IMPROVE AFTER TREATMENT WITH MYCOPHENOLATE MOFETIL}

M McMahon*, B Skaggs, J Grossman, L Sahakian, B Hahn. UCLA David Geffen School of Medicine, Rheumatology, Los Angeles, USA

\subsection{6/lupus-2017-000215.179}

Background and aims Women with SLE have an increased risk of atherosclerosis that is not adequately explained by traditional risk factors. We previously discovered that a "high risk" score on a panel of biomarkers, PREDICTS, confers 28-fold increased odds for carotid plaque in SLE women, and is also associated with IMT progression. The biomarkers included are pro-inflammatory HDL, sTWEAK $\geq 373 \mathrm{pg} / \mathrm{mL}$, homocysteine $\geq 12 \mathrm{mmol} / \mathrm{L}$, leptin $\geq 34 \mathrm{ng} / \mathrm{dL}$, age $\geq 48$ years, and DMII. It is unknown, however, whether these biomarkers are modifiable by SLE disease modifying agents.

Methods This prospective observational study included UCLA cohort patients started on new immunosuppressive agents. Plasma samples were taken at baseline and 12 weeks. HDL antioxidant function was measured by changes in fluorescence intensity of a substrate incubated with LDL and patient HDL. Plasma leptin and sTWEAK were measured using ELISA. Homocysteine was measured in the UCLA clinical labs.

Results 16 subjects started mycophenolate mofetil (MMF), 18 azathioprine (AZA), and 25 hydroxychloroquine (HCQ). In MMF treated subjects, HDL function ( $p=0.009$, paired t-test) and sTWEAK $(p=0.05)$ significantly improved after 12 weeks, while leptin and homocysteine did not significantly change. In HCQ treated subjects, HDL function improved $(p=0.05)$. In the AZA group there were no significant changes in any of the biomarkers. Overall, the mean number of PREDICTS biomarkers at week 12 significantly decreased in the MMF group $(\mathrm{p}=0.03)$.
Conclusions The mean number of "high-risk" cardiac biomarkers significantly improved after initiation of MMF. Further longitudinal studies will determine whether changes in biomarkers reflect decreased cardiovascular events.

\section{PLASMA MYELOPEROXIDASE IS INVERSELY ASSOCIATED WITH FUTURE ATHEROSCLEROSIS PROGRESSION AND INFLAMMATORY HDL FUNCTION IN SLE}

M McMahon*, J Grossman, B Hahn, B Skaggs. UCLA David Geffen School of Medicine, Rheumatology, Los Angeles, USA

\subsection{6/lupus-2017-000215.180}

Background and aims Women with SLE have increased atherosclerosis (ATH) that is not adequately explained by traditional risk factors. We previously discovered that a "high risk" score on a panel of biomarkers, PREDICTS, confers 28-fold increased odds for carotid plaque in SLE women. The biomarkers included in PREDICTS are sTWEAK, pro-inflammatory HDL (piHDL), homocysteine, leptin, age $\geq 48$, and DMII. It is unknown, however, whether other biomarkers of oxidative stress also predict progression of ATH in SLE. The enzyme myeloperoxidase (MPO) catalyses formation of reactive oxygen species and generates piHDL. The aim of this study was to determine whether MPO levels might predict future progression of ATH in SLE.

Methods B-mode and Doppler scanning of carotid arteries was performed at baseline and 24-36 months. Baseline plasma MPO levels were measured using ELISA.

Results Repeat carotid ultrasounds and MPO measurements were completed on 202 SLE women. Plaque progression (defined as new or increased plaque) was seen in 42 subjects (21\%). Baseline MPO levels were significantly lower in SLE patients with plaque progression vs. those without $(\mathrm{p}<0.001)$. Baseline MPO levels were also inversely correlated with piHDL function at follow-up $(\mathrm{r}=-0.33, \mathrm{p}<0.001)$. Using logistic regression, the variables associated with plaque progression in SLE included high PREDICTS (OR 27.0 $\mathrm{p}<0.001$ ), MPO levels in the lowest half (OR 4.2, $\mathrm{p}=0.005$ ), and non-Caucasian ethnicity (OR 4.5, p=0.003).

Conclusions Plasma MPO levels are inversely associated with plaque progression in SLE. Lower baseline MPO levels are also associated with future formation of inflammatory piHDL, suggesting that this could be one mechanism to explain the association.

\section{EPIDEMIOLOGIC PROFILE OF ERECTILE DYSFUNCTION IN SLE: A MULTI-CENTRECENTER STUDY IN LATIN AMERICAN PATIENTS}

IJ Merayo-Chalico*, 'A Barrera-Vargas, 'S Morales-Padilla, 'R Reyna-de-la Garza, ${ }^{1} \mathrm{R}$ Vázquez-Rodríguez, ${ }^{2} \mathrm{M}$ Sotomayor, ${ }^{1} \mathrm{D}$ Gómez-Martín, ${ }^{1} \mathrm{~J}$ Alcocer-Varela, ${ }^{3} \mathrm{I}$ ColungaPedroza, ${ }^{4} \mathrm{C}$ Abud, ${ }^{4} \mathrm{M}$ Martínez-Martínez, ${ }^{5} \mathrm{I}$ Acosta-Hernández, ${ }^{6} \mathrm{C}$ Uriarte-Hernández, ${ }^{7}$ D Fajardo. "Instituto Nacional de Ciencias Médicas y Nutrición "Salvador Zubirán", Immunology and Rheumatology, Mexico City, Mexico; ${ }^{2}$ Instituto Nacional de Ciencias Médicas y Nutrición "Salvador Zubirán", Urology, Mexico City, Mexico; ${ }^{3}$ Hospital Universitario, Rheumatology, Monterrey, Mexico; ${ }^{4}$ Hospital Central, Rheumatology, San Luis Potosí, Mexico; ${ }^{5}$ nstituto Salvadoreño del Seguro Social, Rheumatology, San Salvador, El Salvador; ${ }^{6}$ Hospital Metropolitano Vivian Pellas, Rheumatology, Managua, Nicaragua; ${ }^{7}$ Instituto Mexicano del Seguro Social, Rheumatology, Guadalajara, Mexico

10.1136/lupus-2017-000215.181 